\title{
Chemosensitization of oral squamous cell carcinoma cells to cisplatin by histone deacetylase inhibitor, suberoylanilide hydroxamic acid
}

\author{
HIDEMI RIKIISHI ${ }^{1}$, FUMIAKI SHINOHARA ${ }^{2}$, TOMONORI SATO ${ }^{1,2}$, \\ YOSHITARO SATO ${ }^{1,2}$, MAIKO SUZUKI $^{1}$ and SEISHI ECHIGO ${ }^{2}$ \\ Departments of ${ }^{1}$ Microbiology and Immunology and ${ }^{2}$ Oral Surgery, Tohoku University \\ Graduate School of Dentistry, Aoba-ku, Sendai 980-8575, Japan
}

Received November 30, 2006; Accepted January 18, 2007

\begin{abstract}
In the present study, the precise mechanism of the enhancing action of histone deacetylase (HDAC) inhibitors on cisplatin (CDDP)-induced apoptosis was investigated using suberoylanilide hydroxamic acid (SAHA) in human oral squamous cell carcinoma cells (HSC-3). HDAC inhibitors are promising novel compounds for the treatment of cancer, which cooperate with chemotherapeutic agents to induce apoptosis. Apoptosis enhancement of HSC-3 cells by SAHA was accompanied by the activation of caspase-3, -8 and -9 , suggesting a mitochondrial-dependent amplification loop. Concomitant treatment (CDDP/SAHA) of cells resulted in the most effective enhancement of apoptosis compared to other timing combinations. By means of cell-cycle synchronization, $\mathrm{G}_{0} / \mathrm{G}_{1}$-phase cells proved to be a more sensitive fraction to SAHA action than their synchronized counterparts in other phases. Furthermore, cells treated with SAHA revealed a decrease in intracellular reduced glutathione (GSH) contents. Of importance, the GSH synthesis inhibitor, diethyl maleate, decreased intracellular GSH and enhanced CDDP-induced apoptosis in a similar pattern of timing to SAHA. Thus, SAHA appears to disrupt the intracellular redox balance, which causes maximal apoptosis at the $\mathrm{G}_{0} / \mathrm{G}_{1}$ phase arrested by CDDP in HSC-3 cells. These results demonstrate that HDAC inhibitors not only cause a change in the histone acetylation status, but are also able to influence the apoptotic process at several levels, and GSH plays a key role in governing SAHAdependent enhancement of CDDP-induced apoptosis.
\end{abstract}

Correspondence to: Dr Hidemi Rikiishi, Department of Microbiology and Immunology, Tohoku University Graduate School of Dentistry, 4-1 Seiryo-machi, Aoba-ku, Sendai 980-8575, Japan E-mail: riki@mail.tains.tohoku.ac.jp

Key words: cisplatin, suberoylanilide hydroxamic acid, apoptosis, glutathione, cell-cycle, oral squamous cell carcinoma

\section{Introduction}

The use of cis-diamminedichloroplatinum (II) (cisplatin, CDDP)-based combination chemotherapy remains the standard treatment for oral squamous cell carcinoma; however, for advanced stage cancers, 5-year survival remains dismal, largely because both primary and recurrent cancers often develop resistance to CDDP. As a further complication, resistance to platinum-based compounds frequently leads to resistance to a broad cross-section of other functionally unrelated chemotherapeutic agents. Generally, the malignant phenotype of cancer cells results from a combination of genetic abnormalities and epigenetic modifications throughout acetylation, methylation, and chromatin remodeling, leading to the dysregulation of critical genes controlling cell proliferation, differentiation, and death (1). Attention is currently focused on histone deacetylase (HDAC) inhibitors that represent a new class of targeted anti-cancer agents, either alone or in combination with other chemotherapeutic agents and/or radiation therapy in the treatment of cancer (2). Indeed, we have provided evidence that low doses of HDAC inhibitors, including sodium butyrate $(\mathrm{NaB})$, suberoylanilide hydroxamic acid (SAHA), and MS-275, cooperate with CDDP to induce apoptosis (3). The basic concept is that inhibition of HDAC enzymes relieves gene repression by inducing the hyperacetylation of core histone proteins (2), and several studies have shown altered gene expression upon treatment of cancer cells with HDAC inhibitors (4). Nevertheless, how HDAC inhibitors mediate their diverse effects is not completely understood. Multiple mechanisms have been proposed to explain their anti-cancer activity. For example, acetylation of non-histone proteins is considered an additional mechanism that regulates their apoptotic ability (5). It has been demonstrated that HDAC inhibitors are also involved in the reversible acetylation of non-histone proteins, such as p53, heat-shock protein (Hsp90), tubulin, various transcription factors, and other proteins (6).

Currently available HDAC inhibitors fall into six structural classes. HDAC inhibitors induce differentiation, growth arrest, and apoptosis in cancer cells, whereas they are relatively non-toxic to normal cells (7). SAHA (also known as Vorinostat) 
in phase I/II clinical trials is a prototype of the hydroxamate class, which inhibits class I and II HDAC enzymes with similar potency (5). SAHA has a short half-life, but its effects on the cell are long lasting, with prolonged anti-proliferative response. Contrary to what might be anticipated by the widespread distribution of HDACs in chromatin, inhibition of HDAC activity by SAHA does not cause a global alteration in gene transcription in various transformed cell lines (4). For these reasons, SAHA increased the expression of selected genes involved in cell-cycle regulation, tumor suppression, differentiation, and apoptosis (8). Of note, SAHA has been shown to overcome multi-drug resistance in different cancer cells in vitro and to induce p53-independent apoptosis via the mitochondrial pathway (9). SAHA mediated the cleavage and activation of the pro-apoptotic Bcl-2 family member Bid, resulting in the release of cytochrome $c$ and reactive oxygen species (ROS) from the mitochondria into the cytosol (10).

It is known that a tight chromatin structure prevents CDDP from accessing DNA. One possible strategy to reverse chromatin compaction is the use of chromatin-remodeling compounds. The best characterized compounds are HDAC inhibitors known to uncoil DNA through indirect hyperacetylation of histones. These notions led us to hypothesize that HDAC inhibitors could act as a chemosensitizer for CDDP at several steps. Our findings indicate that using HDAC inhibitors as a sensitizer in chemotherapy-based combination regimens may be a novel strategy to enhance the efficacy of anti-cancer therapy, which warrants further investigation. In addition, the mechanisms for CDDP resistance include the induction of DNA repair enzymes, overexpression of Bcl-2, and increased levels of reduced glutathione (GSH) and associated enzymes. The GSH-based redox system is the best known determinant of CDDP susceptibility. The apoptotic action of CDDP inversely correlates with the endogenous levels of GSH present in a reduced form or activities of GSH-associated enzymes in cancer cells. GSH could combine with CDDP to form a less toxic and more water-soluble glutathione conjugate, bis-(glutathionato)-platinum (11). Conjugation of GSH to CDDP followed by the export of the conjugate by pump appears to be an important resistance mechanism by which cells eliminate CDDP from their cytosol, because the cytotoxicity of CDDP is thought to occur primarily through the induction of DNA damage initially caused by CDDP-DNA adducts (12). In fact, increased concentrations of intracellular GSH are found in cells resistant to CDDP (11). Treatments with agents that experimentally deplete or enhance the GSH content exacerbate or decrease CDDP toxicity, respectively. In this context, it has been showed that the HDAC inhibitor $\mathrm{NaB}$ depletes intracellular GSH in MCF-7 breast carcinoma cells (13). Furthermore, GSH exhibits a large panel of actions in controlling gene expression, apoptosis mechanisms, or membrane transport (14). In particular, the anti-oxidant property of Bcl-2 could be related to GSH because Bcl-2 expression or overexpression increases the GSH level in diverse cells (15). Interesting trials to modulate the GSH level would increase the cancer cell response to CDDP treatment.

In this study, we report that SAHA cooperates in a highly synergistic manner with CDDP to inhibit HDACs and to induce GSH depletion, caspase activation, and cell-cycle specific apoptosis in oral squamous cell carcinoma cells. Based on the biological efficacy of SAHA or CDDP, we tried to analyze the effect of the combination of SAHA/CDDP, and it was anticipated that this therapeutic approach would be effective in oral squamous cell carcinoma cells. These observations may be clinically relevant for the further development of HDAC inhibitors in combination with DNA damaging agents.

\section{Materials and methods}

Materials. We used a commercial preparation of CDDP (Nippon Kayaku, Tokyo, Japan). SAHA was purchased from Alexis Biochemicals (San Diego, CA). Nocodazole and thymidine were obtained from Calbiochem (La Jolla, CA). Caspase inhibitors z-VAD-fmk, DEVD-fmk, IETD-fmk, and LEHD-fmk were purchased from MBL (Nagoya, Japan). Diethyl maleate (DEM) was purchased from Wako Pure Chemicals (Osaka, Japan). Unless indicated otherwise, all other chemicals were obtained from Sigma (St. Louis, MO).

Cell culture. The human oral squamous cell carcinoma cell line HSC-3 (p53-deficient) was kindly provided by the Institute of Development, Aging and Cancer, Tohoku University. HSC-3 cells were grown in RPMI-1640 medium (Nissui Pharmaceutical, Tokyo, Japan) supplemented with $10 \%$ (v/v) heat-inactivated fetal calf serum and $0.2 \%$ sodium bicarbonate and antibiotics in a humidified $5 \% \mathrm{CO}_{2}$ atmosphere at $37^{\circ} \mathrm{C}$.

Cell treatments. Drug treatments consisted of SAHA or CDDP alone or in combination. For combination experiments (CDDP/ SAHA), cells were incubated with drugs at the indicated dose, either concomitantly $(0)$ or sequentially, SAHA $\rightarrow$ CDDP $(-24)$, or CDDP $\rightarrow$ SAHA $(+24)$ for $48 \mathrm{~h}$ of CDDP treatment. For sequential treatments, the first agent was incubated for $24 \mathrm{~h}$, prior to the introduction of the second drug.

Analysis of cell cycling and cell synchronization. Cell-cycle analysis was performed using flow cytometric evaluation of DNA content. Detached cells in the cultures were collected and combined with attached cells that were harvested at the indicated times by trypsinization. Cells were washed in medium, pelleted, resuspended in $70 \%$ ethanol and stored at $-20^{\circ} \mathrm{C}$. Cells were then centrifuged, washed once in PBS, resuspended in PBS with DNase-free RNase and incubated at $37^{\circ} \mathrm{C}$ for $20 \mathrm{~min}$. After incubation, propidium iodide was added to a final concentration of $10 \mu \mathrm{g} / \mathrm{ml}$ and samples were allowed to stand at room temperature for a minimum of $15 \mathrm{~min}$. Cell aggregates were removed by filtration prior to analysis. Cell-cycle analysis was performed on a FACSCalibur flow cytometer (Becton-Dickinson, San Jose, CA). The percentage of cells in each phase of the cell-cycle was determined from the DNA content histograms.

Exponentially growing cells were synchronized to the $\mathrm{G}_{0}$ phase by serum starvation. Cells were maintained in RPMI-1640 medium with $0.2 \%$ serum for 3 days and refed with complete medium containing $10 \%$ serum. Cells were also treated with the specific cell-cycle inhibitors thymidine $(2 \mathrm{mM})$ or nocodazole $(0.5 \mu \mathrm{M})$ for $24 \mathrm{~h}$ to arrest cells in the $\mathrm{S}$ or $\mathrm{G}_{2}$ phase, respectively. Cell-cycle profiles were analyzed every $2 \mathrm{~h}$ after respective treatments by flow cytometry and representative samples were chosen. 
TUNEL assay. TUNEL assay was performed using a Mebstain apoptosis kit from MBL. Cells were collected by centrifugation, washed twice with washing buffer (PBS containing 0.2\% BSA), and fixed in $4 \%$ paraformaldehyde in phosphate buffer on ice for $30 \mathrm{~min}$. Cells were collected, washed with washing buffer, and fixed in $70 \%$ ethanol for $30 \mathrm{~min}$ at $-20^{\circ} \mathrm{C}$. Cells were resuspended in reaction buffer containing FITC-dUTP and terminal deoxynucleotidyl transferase (TdT). Reactions were carried out at $37^{\circ} \mathrm{C}$ for $1 \mathrm{~h}$. After the reactions, cells were washed with washing buffer. FITC-positive cells were counted as apoptotic cells using FACSCalibur.

Assay for HDAC activity. HDAC activity assay was performed using an HDAC colorimetric activity assay kit (Biomol, Plymouth Meeting, PA) following the protocol provided by the supplier. Briefly, total cell lysates were incubated with $0.5 \mathrm{mM}$ colorimetric histone deacetylase lysyl substrate for $30 \mathrm{~min}$ at $37^{\circ} \mathrm{C}$ in a total volume of $50 \mu \mathrm{l}$. After incubation, $50 \mu 1$ of $1 \mathrm{X}$ developer was then added to the sample and incubated at $37^{\circ} \mathrm{C}$ for $15 \mathrm{~min}$. Absorbance was measured at $405 \mathrm{~nm}$ using a colorimetric plate reader.

Measurement of caspase activity. Caspase activity was measured using a colorimetric assay kit from MBL. Synthetic peptides conjugated with $p$-nitroanilide served as substrates for measuring the activity of caspases. In brief, cells were scraped into PBS, pelleted at low speed, and resuspended in lysis buffer for $10 \mathrm{~min}$ at $4^{\circ} \mathrm{C}$. Cell lysates were cleared by centrifugation and assayed for caspase activity using Ac-DEVD- $p$ NA, Ac-IETD- $p$ NA, or Ac-LEHD- $p$ NA peptide substrates, by incubation for $1 \mathrm{~h}$ at $37^{\circ} \mathrm{C}$. Activities were quantified spectrophotometrically at a wavelength of $405 \mathrm{~nm}$. Caspase activity was expressed as the change in absorbance at $405 \mathrm{~nm}$ per mg cell lysate protein. To block caspase activity, the respective specific inhibitors were added at a concentration of $50 \mu \mathrm{M} 1 \mathrm{~h}$ before exposure to CDDP or CDDP/SAHA.

Measurement of GSH levels. To determine the total intracellular GSH content, cells were seeded in dishes, incubated overnight at $37^{\circ} \mathrm{C}$, and then with stimuli. After washing in ice-cold PBS, the cells were harvested in metaphosphoric acid (5\%) by scraping on ice. The remnants obtained were centrifuged at $3,000 \times \mathrm{g}$ for $10 \mathrm{~min}$. The supernatant was collected for reduced GSH measurement using a glutathione assay kit (Calbiochem) with crystalline GSH (Calbiochem) as standard. GSH levels were calculated from a GSH (prepared in 5\% metaphosphoric acid) standard curve and expressed as GSH per mg cell lysate protein.

Statistical analysis. Values are given as the mean \pm SD. Multiple comparisons were performed with the Bonferroni/ Dunn test. $\mathrm{P}<0.05$ was regarded as significant.

\section{Results}

Enhancement of CDDP-induced apoptosis by SAHA. We first investigated whether concomitant administration of SAHA at a low dose affects CDDP-induced apoptosis of HSC-3 cells using flow cytometric analysis following TUNEL staining. Cells were either left untreated, or treated with CDDP $(5 \mu \mathrm{g} / \mathrm{ml})$

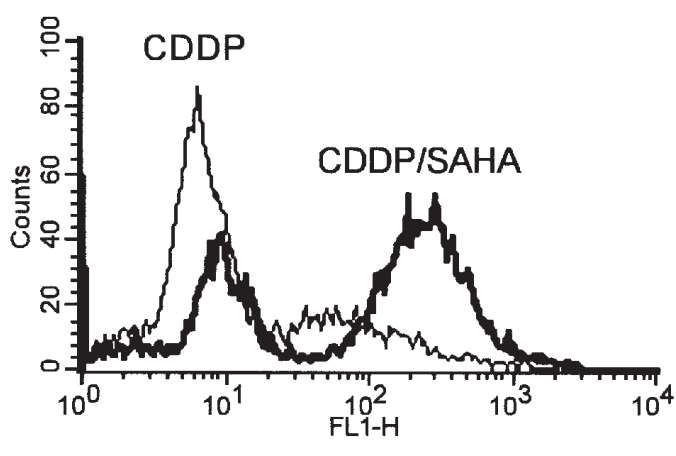

Figure 1. Enhancement of CDDP-induced apoptosis by SAHA. HSC-3 cells were treated with CDDP $(5 \mu \mathrm{g} / \mathrm{ml})$, alone and in combination with SAHA $(1.5 \mu \mathrm{M})$ for $48 \mathrm{~h}$. Cells were then analyzed for apoptosis using a TUNEL assay. Data are representative cytometric profiles and are shown in logarithmic fluorescence intensity.

and/or SAHA $(1.5 \mu \mathrm{M})$ for $48 \mathrm{~h}$. The percentage of apoptotic cells in untreated controls was $4.5 \pm 0.3 \%$. CDDP alone induced $34.3 \pm 1.6 \%$ of apoptosis, while SAHA alone induced $6.1 \pm 0.9 \%$ of apoptosis. Comparing with CDDP alone, the level of CDDPinduced apoptosis was significantly higher for the combination with SAHA $(80.0 \pm 2.7 \%)$. Representative results of flow cytometric analysis are shown in Fig. 1.

Involvement of caspases in the combined effect of CDDP/ $S A H A$. The activation of caspases is a hallmark of apoptosis in many cell types. To determine whether and which caspases are involved in the induction of apoptosis by CDDP/SAHA, cells were exposed to CDDP and/or SAHA for $24 \mathrm{~h}$ and in vitro caspase activity was measured using an enzymatic assay. As shown in Fig. 2A, both CDDP and CDDP/SAHA treatments were associated with significant increased activities of caspase-3. Furthermore, apparent activities of caspase-8 (Fig. 2B) and -9 (Fig. 2C) were detected when treated with this combination (CDDP/SAHA), suggesting the induction of a favorable pre-apoptotic condition. Subsequently, the role of caspases in the apoptotic response to CDDP or CDDP/SAHA was evaluated by using the respective inhibitors (caspase-3, DEVD-fmk; caspase-8, IETD-fmk; or caspase-9, LEHD-fmk) or broad-spectrum irreversible caspase inhibitor (z-VAD-fmk). Pretreatment with $50 \mu \mathrm{M}$ z-VAD-fmk prevented apoptosis induced by CDDP alone and the combination in a highly significant manner. As expected, the role of caspase-3, -8 or -9 activities in apoptosis induced by CDDP or CDDP/SAHA was confirmed by an experiment in which the respective inhibitors positively prevented apoptosis caused by both treatments, although with insufficient inhibition in CDDP/ SAHA treatment (Fig. 2D). Thus, the sensitization of these cells by SAHA was accompanied by the activation of caspase-3, -8 and -9 .

Inhibition of HDAC activity by SAHA. To verify that the SAHA (or CDDP) treatment used here was sufficient to influence HDAC activity, we directly measured HDAC activity by colorimetric assay. Total cell extracts were prepared from cells treated with SAHA $(1.5 \mu \mathrm{M})$ or CDDP $(5 \mu \mathrm{g} / \mathrm{ml})$, and subjected to HDAC activity assay. Although SAHA caused a significant inhibition of HDAC at $4 \mathrm{~h}$, HDAC activity was 

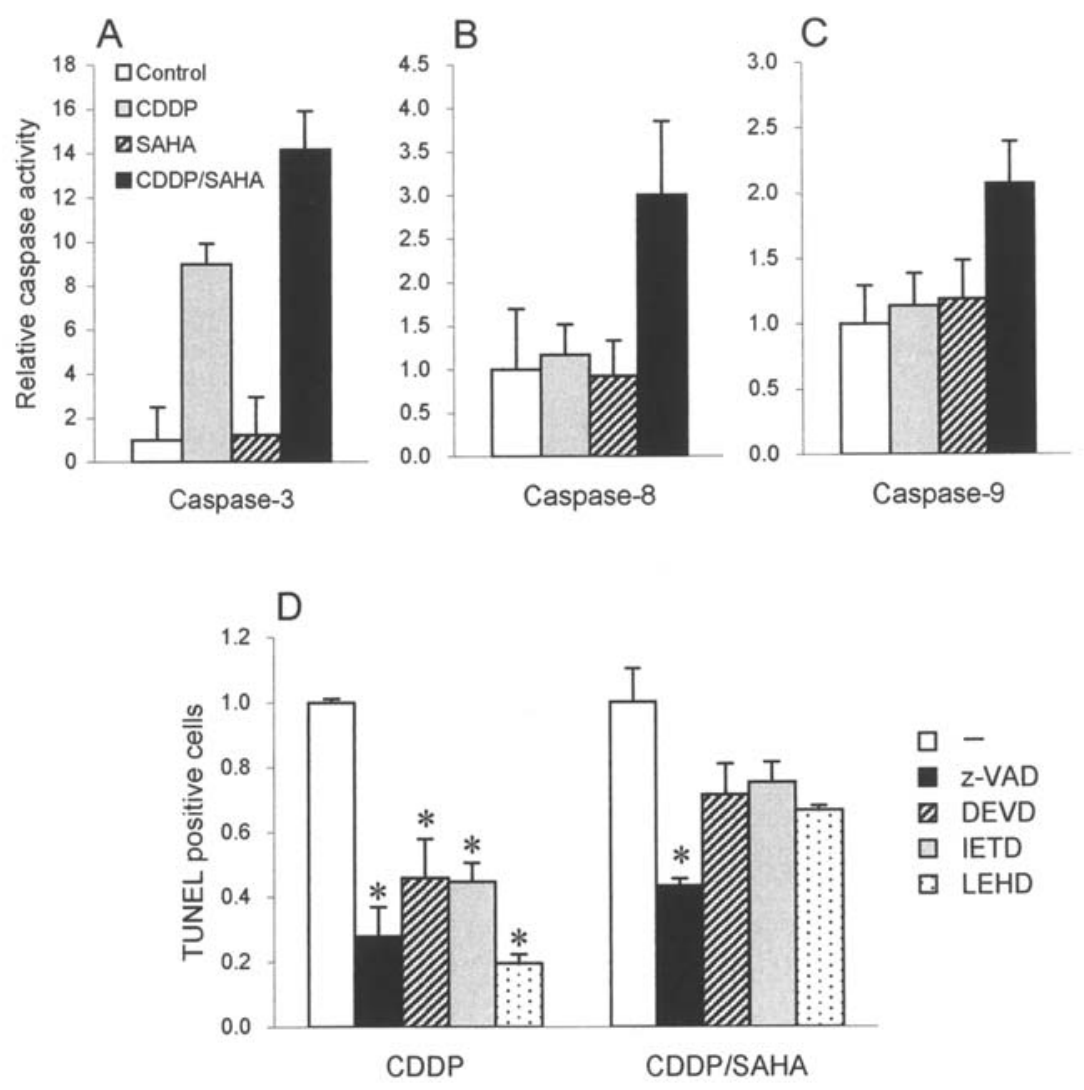

Figure 2. Involvement of caspases in the combined effect of CDDP/SAHA on HSC-3 cells. (A-C) Cells were treated with CDDP (5 $\mu \mathrm{g} / \mathrm{ml}), \mathrm{SAHA}(1.5 \mu \mathrm{M})$, or their concomitant combination for $24 \mathrm{~h}$. After incubation, cell lysates were prepared and quantitatively assayed for respective caspase activities. Results are a representative experiment performed in triplicate. Enzymatic activity is expressed as the relative values to the control group. (D) Cells were pretreated with $50 \mu \mathrm{M}$ caspase inhibitor (z-VAD-fmk, DEVD-fmk, IETD-fmk, or LEHD-fmk) $1 \mathrm{~h}$ before exposure to CDDP or CDDP/SAHA, and incubated for an additional $48 \mathrm{~h}$. Cells were harvested and assayed for apoptosis, and apoptotic cells are expressed as relative values to the control group. Results are expressed as the mean \pm SD of triplicate assays. ${ }^{*} \mathrm{P}<0.05$ vs. control group.
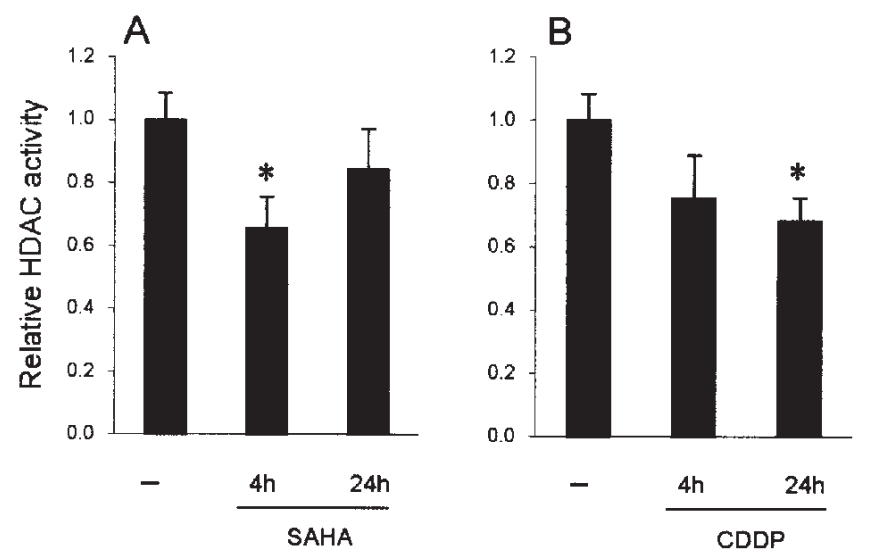

Figure 3. Inhibition of HDAC activity by SAHA or CDDP. HSC-3 cells were treated with $1.5 \mu \mathrm{M}$ SAHA (A) or $5 \mu \mathrm{g} / \mathrm{ml} \mathrm{CDDP} \mathrm{(B)} \mathrm{for} 4$ and $24 \mathrm{~h}$. Total cell lysates were subjected to HDAC activity assay. Absorbance was measured at $405 \mathrm{~nm}$ using a colorimetric plate reader. The absorbance value reflects enzymatic activity. HDAC activity is expressed as relative OD values to the control group. Results show a representative experiment performed in triplicate and are expressed as the mean $\pm \mathrm{SD} .{ }^{*} \mathrm{P}<0.05$ vs. control group.

partially restored at $24 \mathrm{~h}$ (Fig. 3A). Surprisingly, the decrease of HDAC activity was first detected after $4 \mathrm{~h}$ of incubation with CDDP, and maintained over the $24 \mathrm{~h}$ of incubation period in HSC-3 cells (Fig. 3B). It is considered that ROS production by CDDP couples with histone deacetylase inhibition, which might be an important mechanism for inducing apoptosis in cancer cells.

Effects of cell-cycle synchronization on apoptosis triggered by SAHA. In a previous report (3), we suspected that the sequence efficacy of HDAC inhibitors observed might be associated with cell-cycle arrest caused by CDDP. To analyze whether cells of various cell-cycle phases respond differently to SAHA, we synchronized HSC-3 cells at the $\mathrm{G}_{0}$ phase by serum deprivation, or at the $S$ or $G_{2}$ phase by treatment with specific cell-cycle inhibitors, thymidine ( $2 \mathrm{mM})$, or nocodazole $(0.5 \mu \mathrm{M})$ for $24 \mathrm{~h}$, respectively. Fig. $4 \mathrm{~A}$ shows the results of cell-cycle synchronization of cells cultured in different conditions. Flow cytometric analysis revealed that serumdeprived cells were arrested in the $\mathrm{G}_{0} / \mathrm{G}_{1}$ phase of the cellcycle, with very few cells exhibiting DNA content of the $S$ or $\mathrm{G}_{2} / \mathrm{M}$ phase, compared to asynchronous controls or synchronized cells at the $\mathrm{S}$ or $\mathrm{G}_{2}$ phase.

Their respective synchronized cells were further treated for $48 \mathrm{~h}$ with SAHA at a concentration of $15 \mu \mathrm{M}$ that induced significant levels of apoptosis as a single agent in asynchronous controls, reaching $34.6 \pm 7.8 \%$. Significant increases $(56.4 \pm 0.9 \%)$ in the proportion of apoptotic cells were observed when synchronized at the $\mathrm{G}_{0} / \mathrm{G}_{1}$ phase by $72 \mathrm{~h}$ of serum deprivation before SAHA treatment (Fig. 4B). In 

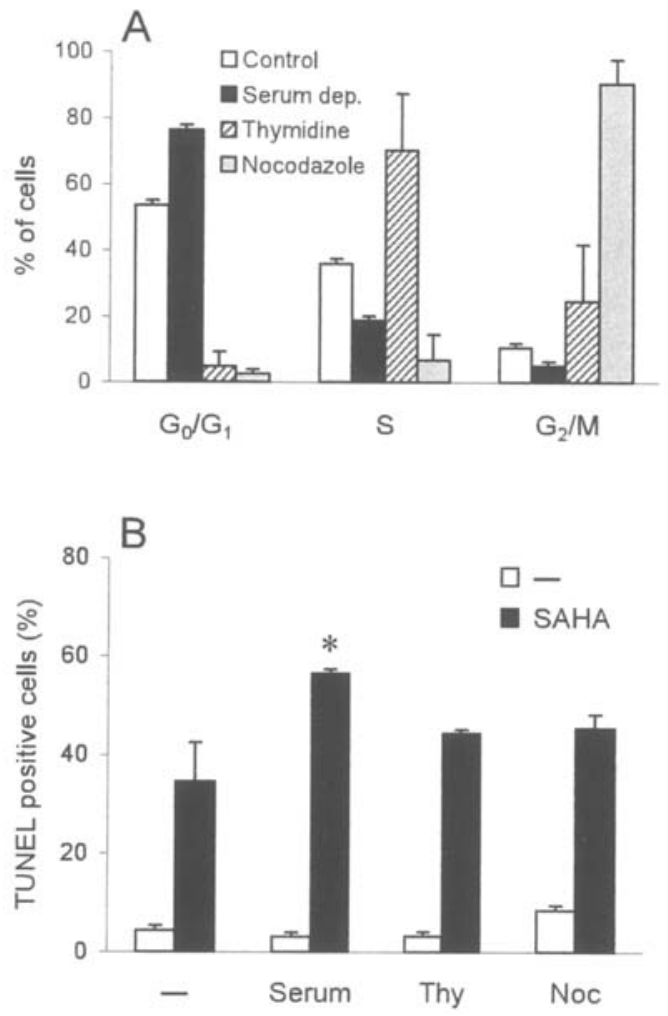

Figure 4. Susceptibility of cells synchronized with the respective cell-cycle phases to apoptosis triggered by SAHA. (A) HSC-3 cells were maintained in medium with $0.2 \%$ serum for 3 days and refed with complete medium containing $10 \%$ serum. Cells were also treated with thymidine (Thy; $2 \mathrm{mM}$ ) or nocodazole (Noc; $0.5 \mu \mathrm{M}$ ) for $24 \mathrm{~h}$, respectively. Cell-cycle distribution was examined with propidium iodide fluorescence by flow cytometric evaluation of DNA content, and cell populations in $\mathrm{G}_{0} / \mathrm{G}_{1}, \mathrm{~S}$, and $\mathrm{G}_{2} / \mathrm{M}$ phases were calculated, respectively. Results are expressed as the percentage of cells in respective phases. (B) Cells were further incubated without or with a high dose of SAHA $(15 \mu \mathrm{M})$ for another $48 \mathrm{~h}$. The percentage of apoptotic cells was determined with a TUNEL assay by flow cytometry. Results are expressed as the mean $\pm \mathrm{SD}$ of triplicate assays. ${ }^{*} \mathrm{P}<0.05$ vs. asynchronous SAHA-treated group.

contrast, pretreatment of thymidine or nocodazole had no significant effects on SAHA-induced apoptosis, unlike under serum deprivation. Thus, cells synchronized at $\mathrm{G}_{0} / \mathrm{G}_{1}$ are more susceptible to SAHA-induced apoptosis than their synchronized counterparts in other phases, suggesting that SAHA may have at least two targets: one that is cell-cycle specific and associated with CDDP-mediated cell-cycle arrest in the $\mathrm{G}_{0} / \mathrm{G}_{1}$ phase and another, unrelated to the cell-cycle, which is responsible for triggering apoptosis indiscriminately and independently of the cell-cycle.

Roles of intracellular GSH in SAHA-mediated enhancement. CDDP may behave as a GSH-sensitive drug in the sense that its toxicity is dependent on the intracellular GSH content (16). Thus, the modulation of GSH level would alter the cell response to apoptosis induced by combined treatment with CDDP/SAHA. To verify the role of intracellular GSH in regulating apoptosis, we monitored the GSH level in HSC-3 cells treated with SAHA or CDDP. As shown in Fig. 5A, there was a gradual decline in the levels of GSH following exposure to $1.5 \mu \mathrm{M}$ SAHA in a time-dependent manner. As CDDP has been shown to be a substrate for the GSH path-

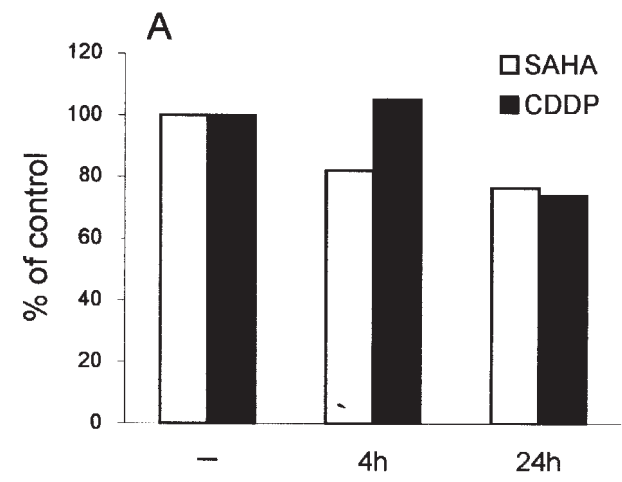

B

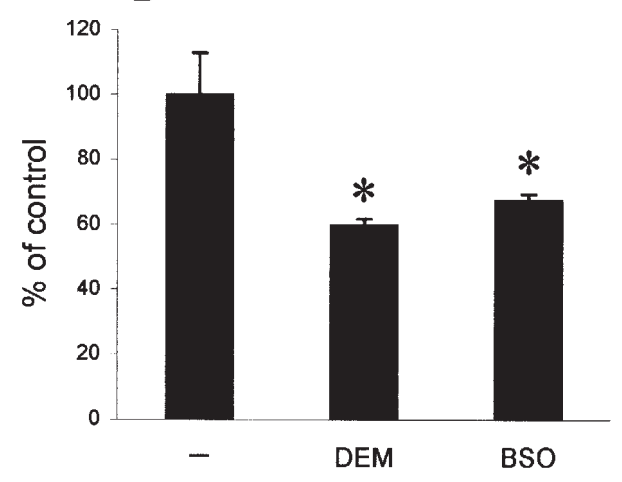

Figure 5. Modulation of intracellular GSH level by SAHA, CDDP, or GSH inhibitors. HSC-3 cells were treated with $1.5 \mu \mathrm{M}$ SAHA or $5 \mu \mathrm{g} / \mathrm{ml} \mathrm{CDDP}$ for 4 and $24 \mathrm{~h}$ (A), or $10 \mathrm{mM}$ DEM or $10 \mathrm{mM}$ BSO for $24 \mathrm{~h}$ (B). Cell lysates were analyzed for intracellular GSH content. The experiment was performed twice, and the results were comparable. The values are expressed as percentage of the GSH level measured in control cells. Results show a representative experiment performed in triplicate and are expressed as the mean \pm SD. ${ }^{*} \mathrm{P}<0.05$ vs. control group.

way, GSH levels after treatment with CDDP for $24 \mathrm{~h}$ were monitored. Treatment with CDDP decreased the intracellular GSH content to some extent (Fig. 5A).

As intracellular GSH contents were significantly decreased in cells after $24 \mathrm{~h}$ of treatment with an inhibitor of GSH synthesis, DEM (10 mM) (Fig. 5B), the importance of GSH depletion as a mediator of apoptosis induction in our experimental conditions was examined using DEM in CDDP-treated cells. As expected, pre-incubation (1 h) with a sub-toxic concentration $(0.5 \mathrm{mM})$ of DEM significantly cooperated with CDDP to induce apoptosis (Fig. 6A). Since DEM alone did not induce apoptosis, it is obvious that DEM is able to augment the potency of CDDP. To our surprise, DL-buthionine$(S, R)$-sulfoximine (BSO), which acts as an irreversible inhibitor of $\gamma$-glutamylcysteine synthetase, failed to increase significantly the susceptibility of HSC-3 cells to CDDP-induced apoptosis, in spite of its ability to lower GSH levels in those cells (Figs. $5 \mathrm{~B}$ and $6 \mathrm{~A})$. We further examined the apoptosis of cells when sequentially treated with DEM before (-24) and after (+24) incubation with CDDP as well as treatment with SAHA in a similar order, compared to the effect on apoptosis induced concomitantly (0) (Fig. 6B). Combined treatment of HSC-3 cells with CDDP/DEM resulted in the similar pattern of apoptotic enhancement to the combination of CDDP/SAHA. Concomitant treatment of cells with either SAHA or DEM resulted in the most effective enhancement of apoptosis 


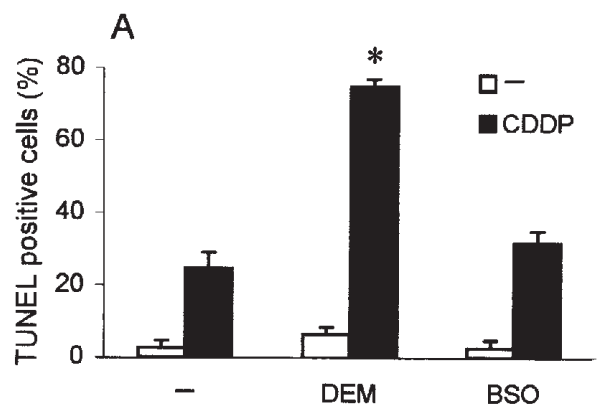

B

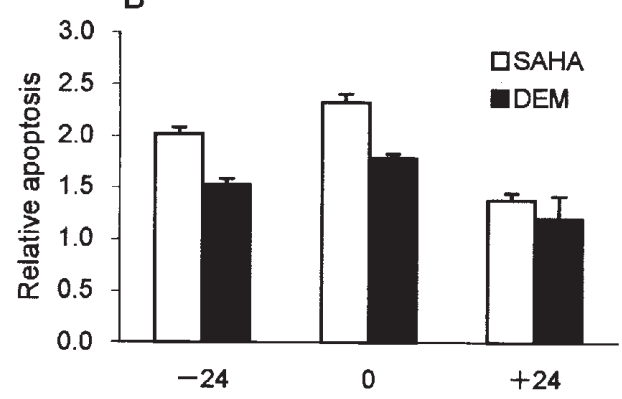

Figure 6. Involvement of intracellular GSH levels in sequential induction of apoptosis. (A) HSC-3 cells were pretreated with DEM $(0.5 \mathrm{mM})$ or BSO $(2 \mathrm{mM}) 1 \mathrm{~h}$ before exposure to CDDP $(5 \mu \mathrm{g} / \mathrm{ml})$, and incubated for an additional $48 \mathrm{~h}$. Cells were harvested and assayed for apoptosis. The percentage of apoptotic cells was determined with a TUNEL assay by flow cytometry. ${ }^{*} \mathrm{P}<0.05$ vs. CDDP alone group. (B) Cells were treated with SAHA $(1.5 \mu \mathrm{M})$ or DEM, concomitantly with CDDP (0), or either for $24 \mathrm{~h}$ before $(-24)$ or $24 \mathrm{~h}$ after (+24) exposure to CDDP. The treatment period of CDDP was $48 \mathrm{~h}$. Cells were then analyzed for apoptosis using a TUNEL assay. Cell apoptosis in each treatment group was calculated as the value relative to CDDP alone. Results are expressed as the mean \pm SD of triplicate assays.

compared to combinations of other orders. Taken together, these results indicated that the depletion of intracellular GSH plays a role in apoptotic enhancement caused by SAHA.

\section{Discussion}

More evidence indicates that a single drug with satisfactory efficacy is rare in cancer therapy and therefore combination therapy using two or more chemotherapeutic agents is one of the most common strategies used in current oncology. Multiple studies of combination chemotherapy, however, have shown limited success with a variable response rate and no significant impact on survival. This draws attention to other partners with the ability to modulate the response of cancer cells to classical chemotherapeutics. With rare exceptions, attempts to combine so-called targeted agents as prevalent partners with standard cytotoxic chemotherapy in advanced cancer have yielded disappointing results. Studies on both laboratory models and in early patient trials have demonstrated that HDAC inhibitors may be a potentially exciting development in cancer therapy (17). Unlike conventional chemotherapeutic agents that often cause DNA damage in both cancer and normal tissues, HDAC inhibitors display strong cancer selectivity and cause less toxicity to normal tissues (7). The synergistic combination of HDAC inhibitors with existing therapeutic agents to reduce drug doses or to overcome drug-resistance phenotypes is yet another strategy that warrants clinical evaluation. In this study, we described the mechanisms of sensitization of oral squamous cell carcinoma cell line HSC-3 to CDDP by the HDAC inhibitor, mainly SAHA. This study is the first demonstration of a connection between the position of cancer cells in the cell-cycle, their cellular GSH redox status, and their apoptotic death, in CDDP/SAHA combination experiments.

Treatment with CDDP alone or in combination with SAHA resulted in a significant increase in the activation of caspase- 3 (Fig. 2A). As the addition of pan-caspase or caspase-3 inhibitor decreased the apoptosis induced by CDDP or CDDP/SAHA (Fig. 2D), caspase-3 could be the actual executive caspase in this experimental system. It is interesting to note that CDDP/ SAHA activated caspase- 8 and -9 in addition to caspase- 3 (Figs. 2B and C) and the use of specific caspase- 8 or -9 inhibitor resulted in decreased apoptosis induced by the combination of CDDP/SAHA (Fig. 2D), suggesting that both CDDP and SAHA are potentially capable of initiating the mitochondrial death pathway directly and inducing cancer cell death under certain conditions $(18,19)$. One study has shown that both caspase- 8 and -9 can be activated downstream of each other in drug-induced apoptosis (20). Caspase-9 has been shown to activate caspase- 8 in a caspase-3-dependent manner $(21,22)$. Caspase- 8 may also activate the mitochondrialdeath pathway and caspase- 9 through the cleavage and translocation of Bid to mitochondria (23). In the end, each agent may be acting through a mitochondrial-dependent amplification loop, as suggested in other cells (24). CDDP/SAHA-induced apoptosis is not completely inhibited by the respective caspase inhibitors, possibly because Bid could continually play a role in the amplification loop. These results support the idea that both caspases are important in determining synergism, and that SAHA possibly lowers the threshold of CDDP-mediated mitochondrial injury and subsequent activation of the caspase cascade.

In a previous report, when cells were exposed to both drugs concomitantly or sequentially, even concomitant treatment and post-treatment with SAHA were effective in apoptotic induction (3). Thus, we speculated that cells arrested in $G_{0} / G_{1}$ by CDDP were vulnerable to the enhancement of CDDPinduced apoptosis by HDAC inhibitors ( $\mathrm{NaB}$ and SAHA). Most anti-cancer drugs that induce apoptosis target the events specific for the cell-cycle, so the analysis of apoptosis with respect to the cell-cycle position is of particular interest. CDDP retards cell-cycle progression by induced accumulation in the $\mathrm{G}_{1}$ phase and the concomitant reduction of cells in the $\mathrm{S}$ phase, respectively, of the cell-cycle in a dose-dependent manner (25). The induction of $\mathrm{p} 21^{\text {waf } 1 / \text { cip } 1}$ altered the progression of the cell-cycle and arrested cells at the $\mathrm{G}_{1}$ phase through blocking cyclin-dependent kinase (CDK4) activity, and promoted the occurrence of apoptosis (26). Exposure to CDDP $(2.5 \mu \mathrm{M})$ for $24 \mathrm{~h}$ enhanced $\mathrm{p} 21^{\text {waf } 1 / \text { cip } 1}$ protein expression in a human non-small cell lung cancer cell line (H1299) unaccompanied by bromodeoxyuridine incorporation, which suggests an arrest in $\mathrm{G}_{1}$ (27). H1299 cells are p53 mutated similar to HSC-3 cells, and the $\mathrm{p} 21$ gene seemed to be regulated by p53-independent factors (28). Attempts at cell-cycle synchronization instead of cell-cycle arrest by CDDP seemed to be a way to demonstrate the selective (apoptotic) effects of SAHA. Serum deprivation 
stress is a commonly used method to synchronize the cell state at the $\mathrm{G}_{0}$ phase of the cell-cycle (29). When cells synchronized at the $\mathrm{G}_{0}$ phase by serum deprivation were treated with the apoptosis-inducible concentration of SAHA and completed medium, a significant increase in apoptotic cells was observed compared to when cells were synchronized at the $S$ or $G_{2}$ phase by treatment with thymidine or nocodazole (Fig. 4). These results suggest that treatment potency with SAHA is at least in part specific to the cell-cycle, and cells in the $\mathrm{G}_{0} / \mathrm{G}_{1}$ phase are more susceptible to SAHA. Likewise, when colon cancer (SW480) and lung cancer (H460) cell lines were examined for their sensitivity to TRAIL after arrest in different cellcycle phases, it was found that the arrest of cells in the $G_{0} / G_{1}$ phase confers significantly higher susceptibility to TRAILinduced apoptosis as compared to cells in the late $G_{1}, S$, or $\mathrm{G}_{2} / \mathrm{M}$ phase (30). It is considered that cell activation from the $\mathrm{G}_{0}$ quiescent stage into the $\mathrm{G}_{1}$ phase and concomitant induction of RNA synthesis significantly increases the efficiency of cell death machinery. A possible explanation for the relative protection of cells in the $S$ or $\mathrm{G}_{2} / \mathrm{M}$ phase might be that the apoptosis machinery of cells in these phases during actual DNA synthesis or cell division is not available to respond to death signals coming from SAHA.

The increase of intracellular GSH levels was shown to accompany the acquisition of cell resistance to CDDP, while the depletion of cellular GSH can sensitize resistant cells to CDDP toxic action (16). GSH is an abundant natural tripeptide found within almost all cells and is transported to the mitochondria, where it plays a protective role as an ROS scavenger (31). Furthermore, GSH binds covalently to CDDP at physiological concentrations and can inhibit the conversion of platinumDNA monoadducts to potentially cytotoxic crosslinks. CDDPGSH conjugates are exported by an ATP-dependent pump. Thus, chemical depletion, physical efflux from the cell, or intracellular redistribution of GSH is associated with the onset of apoptosis in some cell lines. We found that SAHA treatment might cause GSH reduction to increase CDDP sensitivity (Fig. 5A). Furthermore, GSH inhibitors DEM and BSO enhanced the responses to CDDP at different efficiencies (Fig. 6A). The HDAC inhibitor $\mathrm{NaB}$ is known to induce several gene products (glutathione- $S$-transferases and/or glutathione peroxidases) that modify cellular GSH metabolism, and trigger GSH depletion (32). On the other hand, the cyclic depsipeptide FK228, known as a highly potent HDAC inhibitor, has been found to undergo reduction at the disulfide linkage by cellular $\mathrm{GSH}$, resulting in the decrease of GSH, and this reduced product is significantly more potent than the parent compound (33). The sulfhydryl moiety of GSH can be conjugated to electrophilic compounds including some anti-cancer drugs through glutathione- $S$-transferase activities. These results clearly indicate that GSH depletion plays an important role in HDAC inhibitor-induced events of cell-cycle arrest and apoptosis. In addition, GSH depletion might enhance cell death by facilitating the accumulation of ROS (34). CDDP acts as a pro-oxidant agent, and SAHA depletes intracellular GSH, thus suggesting an important role of the GSH level in sensitizing cells to CDDP-induced apoptosis. In addition, the intracellular redox buffering capacity is primarily substantiated by GSH and a dithiol-reducing redox regulatory protein, thioredoxin (TRX). Increased expression of TRX protects cells from apoptosis induced by CDDP in concert with GSH within cells (35). Thioredoxin-binding protein-2 (TBP-2) associates with the active (reduced) form of TRX. Binding of TBP-2 to TRX inhibits thiol-reducing activity and reduces the level of TRX (36). As a candidate for another function of SAHA, it was found that cancer cells (T24, MCF-7, and ARP-1) cultured with SAHA for $15 \mathrm{~h}$ or longer have decreased levels of TRX mRNA, which coincides with the induction of TBP-2 mRNA (37). It is clear that the inhibition of these intracellular redox systems should be taken into account as one mechanism mediating the enhancing effects of SAHA or, conversely, that increased activity of the systems may be a means by which cancer cells can develop a disadvantage for this compound.

As mentioned above, we found that GSH decrease could be responsible for increased apoptosis induced by CDDP/ SAHA. In this study, both DEM and BSO as GSH-depleting agents were used to substantiate apoptotic events in cells $(38,39)$. Concomitant exposure (CDDP/DEM) rendered cells more highly susceptible to CDDP-induced apoptosis than pre- and post-exposure to DEM, whereas BSO exposure had only a small effect on CDDP-induced apoptosis in spite of its ability to lower GSH levels in those cells (Figs. 5B and 6). Although both DEM and BSO are widely used as GSH depleting agents, these agents are known to deplete cellular GSH via different mechanisms (40). DEM is a weak electrophile that inactivates all preformed subcellular pools of GSH by forming a DEM-GSH complex in a reaction catalyzed by glutathione-S-transferase. It has been suggested that DEM administration depletes both cytosolic and mitochondrial GSH pools in vitro, as supported by the profound loss of GSH in treated cells (41). By contrast, it was shown that $\mathrm{BSO}$, a specific inhibitor of $\gamma$-glutamylcysteine synthetase, which is the rate-limiting enzyme in GSH synthesis, does not lower GSH levels in mitochondria because BSO does not penetrate mitochondria. Thus, it is possible that BSO exposure had only a small effect on CDDP-induced apoptosis because mitochondrial GSH synthesis is protected from inhibition by this treatment.

In conclusion, our results indicate that the combination of CDDP with SAHA results in synergistic anti-cancer activity in some types of cancer cells. We have clarified dual mechanisms by which SAHA sensitizes cancer cells to CDDP. One mechanism involves potency by which SAHA treatment is at least in part specific for the cell-cycle, and cells in the $G_{0} / G_{1}$ phase are more susceptible to SAHA. In addition, we have found that SAHA treatment might cause alteration of the cellular GSH status to increase CDDP sensitivity. These important findings could help optimize anti-cancer treatments based on platinum compounds. Possibly of greater potential value is the interaction of a conventional cytotoxic drug (CDDP) and a chemosensitizer (SAHA), thereby allowing the use of less toxic doses of chemotherapy for the treatment of oral squamous cell carcinoma.

\section{Acknowledgements}

We thank Mr. D. Mrozek for editing the manuscript. This work was supported in part by a Grant-in-Aid for Scientific Research 
(16390577) from the Japan Society for the Promotion of Science.

\section{References}

1. Herranz $M$ and Esteller $M$ : New therapeutic targets in cancer: the epigenetic connection. Clin Transl Oncol 8: 242-249, 2006.

2. Lin HY, Chen CS, Lin SP, Weng JR and Chen CS: Targeting histone deacetylase in cancer therapy. Med Res Rev 26: 397-413, 2006.

3. Sato T, Suzuki M, Sato Y, Echigo S and Rikiishi H: Sequencedependent interaction between cisplatin and histone deacetylase inhibitors in human oral squamous cell carcinoma cells. Int $\mathbf{J}$ Oncol 28: 1233-1241, 2006.

4. Glaser KB, Staver MJ, Waring JF, Stender J, Ulrich RG and Davidsen SK: Gene expression profiling of multiple histone deacetylase (HDAC) inhibitors: defining a common gene set produced by HDAC inhibition in T24 and MDA carcinoma cell lines. Mol Cancer Ther 2: 151-163, 2003.

5. Marks PA: The mechanism of the anti-tumor activity of the histone deacetylase inhibitor, suberoylanilide hydroxamic acid (SAHA). Cell Cycle 3: 534-535, 2004.

6. Polevoda B and Sherman F: The diversity of acetylated proteins. Genome Biol 3: 1-6, 2002.

7. Papeleu P, Vanhaecke T, Elaut G, et al: Differential effects of histone deacetylase inhibitors in tumor and normal cells - what is the toxicological relevance? Crit Rev Toxicol 35: 363-378, 2005.

8. Richon VM, Sandhoff TW, Rifkind RA and Marks PA: Histone deacetylase inhibitor selectively induces p $21^{\text {WAF1 }}$ expression and gene-associated histone acetylation. Proc Natl Acad Sci USA 97: 10014-10019, 2000.

9. Ruefli AA, Bernhard D, Tainton KM, Kofler R, Smyth MJ and Johnstone RW: Suberoylanilide hydroxamic acid (SAHA) overcomes multidrug resistance and induces cell death in p-glycoprotein-expressing cells. Int J Cancer 99: 292-298, 2002.

10. Ruefli AA, Ausserlechner MJ, Bernhard D, et al: The histone deacetylase inhibitor and chemotherapeutic agent suberoylanilide hydroxamic acid (SAHA) induces a novel cell death pathway characterized by Bid cleavage and ROS production. Proc Natl Acad Sci USA 98: 10833-10838, 2001.

11. Pompella A, De Tata V, Paolicchi A and Zunino F: Expression of $\gamma$-glutamyltransferase in cancer cells and its significance in drug resistance. Biochem Pharmacol 71: 231-238, 2006.

12. Goodisman J, Hagrman D, Tacka KA and Souid AK: Analysis of cytotoxicities of platinum compounds. Cancer Chemother Pharmacol 57: 257-267, 2006.

13. Louis M, Rosato RR, Brault L, et al: The histone deacetylase inhibitor sodium butyrate induces breast cancer cell apoptosis through diverse cytotoxic actions including glutathione depletion and oxidative stress. Int J Oncol 25: 1701-1711, 2004.

14. Hammond CL, Lee TK and Ballatori N: Novel roles for glutathione in gene expression, cell death, and membrane transport of organic solutes. J Hepatol 34: 946-954, 2001.

15. Wright SC, Wang H, Wei QS, Kinder DH and Larrick JW: Bcl-2mediated resistance to apoptosis is associated with glutathioneinduced inhibition of AP24 activation of nuclear DNA fragmentation. Cancer Res 58: 5570-5576, 1998.

16. Siddik ZH: Cisplatin: mode of cytotoxic action and molecular basis of resistance. Oncogene 22: 7265-7279, 2003.

17. Kelly WK, O'Connor OA and Marks PA: Histone deacetylase inhibitors: from target to clinical trials. Expert Opin Investig Drugs 11: 1695-1713, 2002.

18. Alas S, Ng CP and Bonavida B: Rituximab modifies the cisplatinmitochondrial signaling pathway, resulting in apoptosis in cisplatin-resistant non-Hodgkin's lymphoma. Clin Cancer Res 8: 836-845, 2002.

19. $\mathrm{Xu} \mathrm{W}$, Ngo L, Perez G, Dokmanovic M and Marks PA: Intrinsic apoptotic and thioredoxin pathways in human prostate cancer cell response to histone deacetylase inhibitor. Proc Natl Acad Sci USA 103: 15540-15545, 2006.

20. Wieder T, Essmann F, Prokop A, et al: Activation of caspase-8 in drug-induced apoptosis of B-lymphoid cells is independent of CD95/Fas receptor-ligand interaction and occurs downstream of caspase-3. Blood 97: 1378-1387, 2001.

21. Slee EA, Harte MT, Kluck RM, et al: Ordering the cytochrome c-initiated caspase cascade: hierarchical activation of caspases-2, $-3,-6,-7,-8$, and -10 in a caspase-9-dependent manner. J Cell Biol 144: 281-292, 1999.
22. Sun XM, MacFarlane M, Zhuang J, Wolf BB, Green DR and Cohen GM: Distinct caspase cascades are initiated in receptormediated and chemical-induced apoptosis. J Biol Chem 274: 5053-5060, 1999

23. Suliman A, Lam A, Datta R and Srivastava RK: Intracellular mechanisms of TRAIL: apoptosis through mitochondrialdependent and -independent pathways. Oncogene 20: 2122-2133, 2001.

24. Henderson C, Mizzau M, Paroni G, Maestro R, Schneider C and Brancolini C: Role of caspases, Bid, and p53 in the apoptotic response triggered by histone deacetylase inhibitors trichostatinA (TSA) and suberoylanilide hydroxamic acid (SAHA). J Biol Chem 278: 12579-12589, 2003.

25. Wang G, Reed E and Li QQ: Molecular basis of cellular response to cisplatin chemotherapy in non-small cell lung cancer (Review). Oncol Rep 12: 955-965, 2004.

26. Meng LH, Zhang H, Hayward L, Takemura H, Shao RG and Pommier Y: Tetrandrine induces early $\mathrm{G}_{1}$ arrest in human colon carcinoma cells by down-regulating the activity and inducing the degradation of $\mathrm{G}_{1}-\mathrm{S}$-specific cyclin-dependent kinases and by inducing p53 and p21 ${ }^{\mathrm{Cip} 1}$. Cancer Res 64: 9086-9092, 2004.

27. Crescenzi E, Chiaviello A, Canti G, Reddi E, Veneziani BM and Palumbo G: Low doses of cisplatin or gemcitabine plus Photofrin/photodynamic therapy: disjointed cell cycle phaserelated activity accounts for synergistic outcome in metastatic non-small cell lung cancer cells (H1299). Mol Cancer Ther 5: 776-785, 2006.

28. Agami R and Bernards R: Distinct initiation and maintenance mechanisms cooperate to induce G1 cell cycle arrest in response to DNA damage. Cell 102: 55-66, 2000.

29. Kues WA, Anger M, Carnwarth JW, Motlik J and Nieman H: Cell cycle synchronization of porcine fibroblasts: effects of serum deprivation and reversible cell cycle inhibitors. Biol Reprod 62: 412-419, 2000.

30. Jin Z, Dicker DT and Deiry WS: Enhanced sensitivity of G1 arrested human cancer cells suggests a novel therapeutic strategy using a combination of simvastatin and TRAIL. Cell Cycle 1: $82-89,2002$.

31. Lluis JM, Morales A, Blasco C, et al: Critical role of mitochondrial glutathione in the survival of hepatocytes during hypoxia. J Biol Chem 280: 3224-3232, 2005.

32. Louis M, Rosato RR, Battaglia E, et al: Modulation of sensitivity to doxorubicin by the histone deacetylase inhibitor sodium butyrate in breast cancer cells. Int J Oncol 26: 1569-1574, 2005.

33. Furumai R, Matsuyama A, Kobashi N, et al: FK228 (depsipeptide) as a natural prodrug that inhibits class I histone deacetylases. Cancer Res 62: 4916-4921, 2002.

34. Dickinson DA and Froman HJ: Cellular glutathione and thiols metabolism. Biochem Pharmacol 64: 1019-1026, 2002.

35. Powis G and Montfort WR: Properties and biological activities of thioredoxins. Annu Rev Pharmacol Toxicol 41: 261-295, 2001.

36. Nishiyama A, Matsui M, Iwata S, et al: Identification of thioredoxin-binding protein-2/vitamin $\mathrm{D}(3)$ up-regulated protein 1 as a negative regulator of thioredoxin function and expression. J Biol Chem 274: 21645-21650, 1999.

37. Butler LM, Zhou X, Xu WS, et al: The histone deacetylase inhibitor SAHA arrests cancer cell growth, up-regulates thioredoxin-binding protein-2, and down-regulates thioredoxin. Proc Natl Acad Sci USA 99: 11700-11705, 2002.

38. Coffey RN, Watson RW, Hegarty NJ, et al: Thiol-mediated apoptosis in prostate carcinoma cells. Cancer 88: 2092-2104, 2000.

39. Takahashi M, Sato T, Shinohara F, Echigo S and Rikiishi H: Possible role of glutathione in mitochondrial apoptosis of human oral squamous cell carcinoma caused by inorganic selenium compounds. Int J Oncol 27: 489-495, 2005.

40. Yang X, Wu X, Choi YE, Kern JC and Kehrer JP: Effect of acrolein and glutathione depleting agents on thioredoxin. Toxicology 204: 209-218, 2004.

41. Garcia-Ruiz C, Colell A, Morales A, Kaplowitz N and Fernandez-Checa JC: Role of oxidative stress generated from the mitochondrial electron transport chain and mitochondrial glutathione status in loss of mitochondrial function and activation of transcription factor nuclear factor- $\kappa \mathrm{B}$ : studies with isolated mitochondria and rat hepatocytes. Mol Pharmacol 48: 825-834, 1995. 